\title{
Basics of evaluation methods of HPP resource usage during operation in IPS
}

\author{
Alyona V. Sidorova \\ Novosibirsk State Technical University, \\ Power Engineering Faculty \\ Russia \\ sidorovaa94@mail.ru \\ Anna Y. Arestova \\ Novosibirsk State Technical University, \\ Power Engineering Faculty \\ Russia \\ ann.arestova@gmail.com
}

\author{
Anastasia G. Rusina \\ Novosibirsk State Technical University, \\ Power Engineering Faculty \\ Russia \\ rusina@corp.nstu.ru \\ Tamara A. Filippova \\ Novosibirsk State Technical University, \\ Power Engineering Faculty \\ Russia \\ filippova@corp.nstu.ru
}

\begin{abstract}
In the electric-power systems, which include hydroelectric power plants (HPP), the important objective is the increasing of water resources utilization efficiency. Hydroelectric power plants have a great impact on the distribution of power at the regional level and especially in the wholesale electric power market in models of the type «hydro resources - consumption of electric power». Some principles of calculation methodology for HPP are set forward in this paper. The authors call this type of model R-P-C (resources, production, capacity) of HPP. Taking into consideration the uncertainty of HPP consumption and information difficulties, tasks are traditionally divided into two parts (depending on the period of planning): the long-term and short-term modes. In this paper, the authors introduce the original methodology of evaluation of HPP's resources usage. The methodology consists of five units. The given methodology can serve as a basis for making a program for simulation of interconnected power system modes.
\end{abstract}

Keywords- HPP; water energy resources; hydroelectric mode; structural model; utilization efficiency; technologic process; energy balance.

\section{INTRODUCTION}

Hydroelectric power plants have a great impact on the distribution of power at the regional level and especially in the wholesale electric power market in models of the type "hydro resources - consumption of electric power". Some principles of methodology of calculations for the HPP are set forward in this paper. The authors call this type of model R-P-L (resources, production, capacity) HPP. Taking into consideration the uncertainty of HPP flow and information difficulties, tasks are traditionally divided on two parts (depending on the period of planning), the long-term and the short-term modes. [1,2,3]. In the scheme of using an R-P-L model, there are a lot of features and changes compared to previous developments, made for the UES (united energy system) by the USSR $[2,4,5]$.

\section{MATERIALS AND METHODS, THEORY}

\section{A. Features of algorithmization of the RPL (Resources Production Load) model}

Unit 1 - information space. A fundamental issue here is a necessity to use hydroenergy and hydraulic information on pressures, flow, charges, levels of ponds, which increases not only the size of arrays of traditional information of thermal interconnected power systems, but also the uncertainty of all tasks of planning modes of hydrothermal interconnected power systems. The unit includes the prediction models because without forecasts, it is impossible to solve the modal problem of the interconnected power system.

Unit 2 - hydropower mode. The optimal use of water resources of HPP is determined (the usage of the domestic supply and inventory flow in the reservoir). The main task is the use of hydropower resources and getting the maximum power generation. This means that it is required to obtain the maximum efficiency from the pressure and flow rate. Optimization is done depending on the criterion of making the electric power maximum for the period of the complete cycle of hydropower calculations, in other words for a hydrological year. This corresponds to the condition of the late reservoir drawdown during the low water and to the early filling of the reservoir during the flood. If one considers a cascade of hydroelectric power plants, the criterion of maximum pressure of underlying cascade plants must be added. All the foregoing corresponds to the rational conduct of business and a maximum of sales of electric energy of HPP and optimizations of the mode of the interconnected power system.

In the unit, one solves a complex of problems considering the rational use of hydropower resources of single and connected plants in the cascade. Electricity generation at the HPP depends on the flow, and use of flow mode, essentially depends on the head flow. The main role is played by the 
criterion of power generation optimization. Criterion $\ni_{H P P} \rightarrow \max$ is the easiest as it does not require the analysis of the use of hydroelectric power plants in the system, but the solution is certainly not the best.

Depending on the purpose of the calculation, if there is insufficient information about the loads in the nodes, the simulation of the cascade can be performed by one equivalent node, as shown in Figure 1:

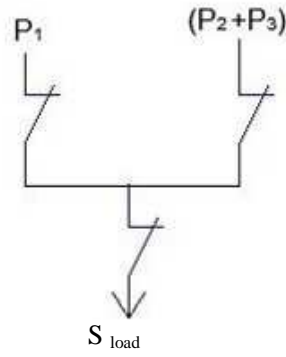

Fig. 1. Modeling of the HPP cascade using an equivalent node

If there is some electrical model of linking the plants of the cascade, then the calculation is made using the model shown in Figure 2:

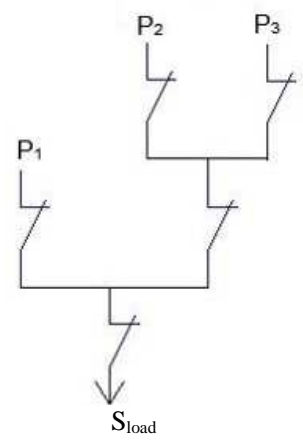

Fig. 2. Modeling of the HPP cascade by means of a separate representation of each plant

It should be noted that for these schemes, a system of equations for the balance of power and energy was compiled, which allows us to describe the mode of operation of the plants in the cascade and to establish communication in the hydraulic and electric modes.

Unit 3 - technological process of the HPP. In it, the tasks of in-plant solutions of the choice of the composition and the mode of hydraulic generators with the preset loading plant by the criterion of maximum efficiency are considered. This is the main indicator of the process of the transformation of potential energy into the working one and the accomplishment of criterion $\ni_{H P P} \rightarrow \max$.

HPP efficiency is the multiplication of the efficiency of the pressure $(\mathrm{H})$, flow rate $(\mathrm{Q})$ and the technological process:

$$
\eta_{H P P}=\eta_{H} \cdot \eta_{Q} \cdot \eta_{\text {tech }}
$$

$$
\begin{gathered}
N_{\text {input }}=k \cdot Q \cdot H \\
\eta_{H}=\frac{H_{\text {useful }}}{H_{\text {input }}}=\frac{H_{\text {input }}-\Delta H}{H_{\text {input }}} \\
\eta_{Q}=\frac{Q_{\text {useful }}}{Q_{\text {input }}}=\frac{Q_{\text {input }}-\Delta Q}{Q_{\text {input }}} \\
\eta_{\text {tech }}=\frac{N_{\text {useful }}}{N_{\text {input }}}=\frac{N_{\text {input }}-\Delta N}{N_{\text {input }}}
\end{gathered}
$$

where: $\Delta H, \Delta Q$ and $\Delta N$ indicate the loss of pressure, flow and power of HPP, or variability of these factors affecting the efficiency.

The full efficiency of the turbine installation by the water pressure head flow is:

$$
\begin{gathered}
\eta_{H}=\frac{H_{\text {useful }}}{H_{\text {useful }}-\Delta H_{d s}-\Delta H_{\text {w.f. }}-\Delta H_{t u r b}-\Delta H_{\text {a.f. }}} \\
=\eta_{H d s} \cdot \eta_{H w . f .} \cdot \eta_{H t u r b} \cdot \eta_{H a . f .}
\end{gathered}
$$

where the indices represent:

$d s$ - downstream;

w.f. - water-supply facilities;

turb - turbine;

a.f. - allocating facilities.

Unit 4, 5 - HPP mode in the IPS and the commercial balance. In the interconnected power system, the condition of receipt of a minimum of expenses must be observed, taking into account the structure of powers of the interconnected power system [6,7]. The main features of the interconnected power system correspond to its energy balance, and the criterion of optimization is to minimize the total costs of all units (plants and networks). Such optimization is produced for a calendar year with the change of loading.

In many works, it is shown that the mode with a criterion of maximum power generation does not always correspond to the mode with a criterion of minimum expenses; moreover, the result in the first case is a few percent worse. Therefore, the criterion of minimum expenses was always in favor. However, now it is connected with large information difficulties and there are two alternatives: either the mode is calculated for the plant (in this case the system information is required), or the mode is calculated for the system and then hydrological information is needed.

It is reasonable to perform this calculation by the scheme of the successive specification of the mode. First, the mode is calculated for the HPP by the criterion of maximum energy production. The second stage is its after-optimization by the criterion of minimal costs. And finally, there is a third stage - 
by the maximum of sales profit in the electricity market. Of course, in this case, there will be some loss of efficiency and the use of potential resources, but this approach removes a part of the information problems.

\section{B. Fuel effectiveness of resources when using HPP - a factor} of great importance for the interconnected power system

The concept of fuel effectiveness reflects the impact of the HPP on the energy balance of the interconnected power system, as it is obvious that the HPP always gives some benefit to the system - and especially for the mode of the thermal stations [1]. In the power balances, hydroelectric power plants are placed mostly without taking into consideration the effectiveness of power resources.

The fuel effectiveness of water resources (consumption of water and the flowing) depends mainly on the indexes of the energy balance of powers of the interconnected power system. The power balance changes every day, and by seasons; accordingly, the fuel effectiveness of water resources also changes. For determination of fuel effectiveness, the characteristic of relative cumulative gains of the interconnected power system, power balance data, and plant locations on the balance sheet (peak, half-peak, base) is used.

At that, the relative cumulative gains in fuel and costs change:

$$
\frac{\Delta B}{\Delta P}, \frac{\Delta H}{\Delta P}
$$

Fuel effectiveness can change the content and the structure of the cost of HPP electric power:

$$
C=C_{\text {cond.perm. }}+C_{\text {res.water }}=C_{\text {cond.perm. }}+\frac{\Delta U}{\Delta P} \cdot P_{H P P}
$$

Component $C_{\text {res.water }}=\frac{\Delta H}{\Delta P} \cdot P_{H P P}$ can be significant and allows us to consider many modal interconnected power system objectives, taking into account the value of water resources.

It is necessary to take into account the effectiveness of the load distribution among the interconnected power system stations. Such problem has been worked out in detail.

The full algorithm includes 5 main units listed above: information, hydropower, technological, commercial, and the unit of energy balances of the hydrothermal interconnected power system. The calculation of the mode is conducted, starting from a base original plan to a final one, thus the HPP mode is calculated consistently by three criteria: the maximum power generation, minimum costs, maximum profit.

There are indicators of the evaluation of HPP modes in the R-P-L model. Nowadays, taking into account the interests of the business, the main and almost the only indicator is the profit and price. The fallacy of this is shown in many studies, for example, in $[6,8,9]$.
The indexes of effectiveness of the hydroelectric power plants can be calculated with different aims, and their form and content depends on it. Without exception, all effectiveness indicators of the work of the HPP depend on its power generation; it also requires the solution of many problems.

The structural model of production of electric power at HPP must take into account the entire process: from the resources of production to realization of electric power across all types of activity.

\section{The use of electricity resources}

The hydropower potential depends on the parameters of the plant, the flow of the river, the mode of consumption of water resources. Electricity generation is determined by calculations based on the data of water resources, on head flow modes, on the efficiency of the technological process of the plant. All components are variable in time and have a high level of uncertainty.

The transport of the electric power and the mode of transmission of electric power by the network companies of $\mathrm{NC}$ can have different scales and economic forms [5]. Network companies and enterprises, engaged in transport of electricity, are the resellers and the price of all enterprises will be:

$$
\bigsqcup_{\text {trans }}=\Sigma Ц_{\text {transi }}
$$

Usually HPP has no freedom in choice of routes of the transport and indisposes of the data on their determination. Then the development of the methods of creating an electrical scheme or a special prediction methodology for estimating the costs of transport is needed. HPP can solve independently some transporting tasks.

\section{CAClCUlations}

The methodology of calculation of indicators and evaluation of HPP activities consists in the following. The scopes of this paper do not allow considering methodology of calculation of indexes of effectiveness in detail, but it is obvious that it is unlikely to be realized without a special algorithm. Let us give some fragments of this technique.

Let us consider the concept of meaningful efficiency by the head flow (water pressure) which requires specification of the concepts of supplied head water and useful head pressure:

$$
\eta_{H}=\frac{H_{\text {useful }}}{H}
$$

Pressure of the block for a dam HPP is:

$$
H_{b l}=Z_{\text {upstream }}-Z_{\text {downstream }}
$$

For derivation HPP, the pressure is:

$$
H_{b l}=Z_{\text {upstream }}-Z_{\text {downstream }}-\Delta H_{\text {der }}
$$


The pressure, supplied to the turbine, is less than the unit pressure by the value of the losses in water-supply facilities, which can be calculated in advance:

$$
H_{\text {sup.turb. }}=H_{b l}-\Delta H_{w . f} \text {. }
$$

Useful pressure of the turbine installation is less than the unit pressure by the size of losses of pressure in the turbine and in lateral facilities:

$$
H_{\text {useful }}=H_{\text {sub.turb. }}-\Delta H_{\text {turb }}-\Delta H_{\text {a.f. }} .
$$

Overall efficiency of the turbine installation by the pressure is:

$$
\begin{gathered}
\eta_{H}=\frac{H_{\text {useful }}}{H} \\
\eta_{H}=\frac{H_{\text {useful }}}{H_{\text {useful }}-\Delta H_{d s}-\Delta H_{\text {w.f. }}-\Delta H_{\text {turb }}-\Delta H_{\text {a.f. }}}= \\
=\eta_{H d s} \cdot \eta_{H w . f .} \cdot \eta_{H t u r b} \cdot \eta_{H a . f .}
\end{gathered}
$$

Over time, the pressure characteristics change.

Flow efficiency can be calculated by the upstream, and for a cascade - by the downstream.

The equation of balance looks like:

$$
\begin{aligned}
Q_{w b}(t) & =Q_{\text {in }}(t) \pm Q_{i}(t)+Q_{p r}(t)-Q_{\text {surf }}(t) \pm \\
& \pm Q_{\text {l.ice. }}(t)-Q_{\text {l.filtr. }}(t)-Q_{\text {in }}(t)
\end{aligned}
$$

where:

$Q_{\text {in }}$ - inflow;

$Q_{i}$ - inventory reservoir flow;

$Q_{p r}$ - precipitation;

$Q_{\text {surf }}$ - evaporation from its surface;

$Q_{\text {l.ice. }}$ - ice formation losses;

$Q_{\text {l.filtr. }}$ - filtration losses through reservoir bed;

$Q_{i n}$ - water intake for the needs of water consumers.

The balance of the costs in the downstream plant is:

$$
\begin{gathered}
Q_{\text {downstream }}(t)=Q_{H P P}(t)+Q_{\text {s.disch. }}(t)+ \\
+Q_{\text {lock }}(t)+Q_{\text {filtr }}(t)+Q_{\text {other }}(t)
\end{gathered}
$$

where:
$Q_{H P P}$ - the water flow through HPP turbines;

$Q_{\text {s.disch. }}$ - consumption of single discharges through spillways;

$Q_{\text {lock }}$ - locking consumption;

$Q_{\text {filtr }}$ - water filtration through hydrotechnical buildings and different devices of the plant;

$Q_{\text {other }}$ - consumption of water that enters the lower pool through the fish passes, ice passes and other facilities.

The balance of charges by the plant is:

$$
Q_{\text {upstream }}(t)=Q_{\text {downstream }}(t)
$$

Mostly, components $Q_{p r}, Q_{\text {surf }}, Q_{\text {l.ice. }}, Q_{\text {l.filtr }}, Q_{\text {in }}$ are recorded in the form of corrections to the inflow of household, and then the balance equation is written in the standard form:

$$
Q_{\text {downstream }}(t)=Q_{H P P}(t) \pm Q_{\text {s.disch. }}(t)
$$

From these equations, it is easy to determine the factors, affecting the efficiency of the flow rate.

The efficiency of the technological process includes the transformation of supplied energy in the working bodies of the turbine generator and water-supply constructions.

Power $N_{\text {input }}$ is supplied, power on the clamps of generator will be $N_{f u l l}$, and the efficiency of the aggregate unit is:

$$
\eta_{a}=\eta_{t} \cdot \eta_{g} \cdot \eta_{w \cdot f}
$$

where the indexes are: $\eta_{t}$ - turbine, $\eta_{g}$ - generator, and $\eta_{w . f}$. - efficiency of the water-supply constructions.

In conclusion, it must be emphasized that a special technique is required for determining all the components of factors and efficiency, not the starting but actual conditions. This is a difficult information task.

The cost of HPP electric energy is calculated in the following way. Specific production costs at an operating HPP:

$$
C_{H P P}=\frac{U_{\text {cond.perm. }}}{Э_{H P P}}=\frac{{U_{\text {depr }}}+H_{f . c .}+H_{\text {other }}}{Э_{H P P}}
$$

where:

$$
U_{\text {cond.perm. }}-\text { contingent fixed costs; }
$$

$U_{d e p r}$ - depreciation costs; 
$U_{f . c .}{ }^{- \text {fixed costs; }}$

$U_{\text {other }}$ - other costs.

Economic efficiency of the HPP is a minimum cost. Its size depends on the power generation. The most important fact is that the sales price of electricity is determined in direct proportion to the prime cost.

Commercial effectiveness and address estimations of electric power are as follows. The wholesale electricity market operates ин the rules set by the market organizers. They are not coordinated with the technological process of transformation of water power at the different stages. At the same time, one can not exclude this stage in the complex estimation of the effectiveness of using the HPP resources [4, $5,9,10]$.

\section{RESULTS}

Let us name the indicators reviewed in this work. The primary indicator is the maximum generation electric power of the HPP, which is achieved due to optimization of: water pressure, flow, efficiency of equipment and technology, fuel effectiveness, load distribution in the interconnected power system, losses of electric power in networks, commercial advantages in the relations of purchase/of sale. In the power system of power generation on HPP is depends on the function of other plants, control and cost analysis system management.

\section{A. The effectiveness of the use of the hydroelectric power plants in the complex}

The effectiveness includes two components - the expenses and the benefits. The expenses are the prime cost of $C_{H P P}$, benefit is a modal effectiveness, which the HPP yields while working in interconnected power system and the commercial effectiveness, which the HPP receives as the subject of the electro energy market.

Then, the total effectiveness will be:

$$
S=k_{c} C-\underset{j}{\left(\sum k_{j} \Delta \ni_{e f . j}\right)}
$$

The indicators of effectiveness may have different units of measurement of efficiency in percent's, the energy in $\mathrm{kWh}$, the prime cost in rubles and they should be lead to a single form using $\mathrm{k}$ reduction factors.

\section{B. The practical importance of performance indicators}

The practical importance of performance indicators are the following practical areas of application performance are the most possible:

1) Complex efficiency allows one to compare the plants against each other and to compare them to the norms on the whole.

2) The comparison is possible both on the separate stages of the use of water resources and on the separate tasks.

3) To analyses and set the ways of energy-savings.

\section{CONCLUSION}

The presented methodology concerning the effectiveness of the HPP use, starting from the resources and ending with the commercial results, is up-to-date both for the operating plants and for the designed ones. Nowadays, new HPPs are supposed to be planned and created, and this methodology influences an investment project.

The estimation of efficiency of using water resources is relevant for prices in the electric power engineering market as well.

Let us give an example for IPS Siberia, in the balances of which Novosibirsk, Krasnoyarsk, Bratsk, Ust - Ilim, Sayano Shushenskaya and Irkutsk hydroelectric power plant participate. They have different water pressure from about 20 to $200 \mathrm{~m}$. A unit of streamflow volume at Novosibirsk HPP has about 10 times less potential energy than the flow at Krasnoyarsk HPP and 20 times less than at SayanoShushenskaya HPP - with little difference in energy prime costs. And the plants base only on the prime cost having their price requests. The potential is practically ignored at the plant, and it is unknown even on IPS of Siberia.

For the operated HPP it is important to estimate their effectiveness correctly not only on the stated prices, but with the consideration of their connection of prices with their way of the use of resources: renewable and cheap, water resources are the national treasure. The topic reviewed in the work requires a deep research.

\section{References}

[1] T.A. Filippova, M. Sh. Misrikhanov, Y.M. Sidorkin, A.G. Rusina, Hydropower: Proc. Benefit - Ed. 3rd, Revised, 2013, p. 620.

[2] V.I. Obrezkov, Hydropower: A Textbook for high schools, Ed. 2nd, 1988, p. 512.

[3] Z.M. Gafarova, "Regional problems of efficient use of water and energy resources," Science, new technologies and innovations, №5, pp. 104107, 2015.

[4] P.M. Erokhin, K.S. Kolyasnikov, "Optimization of long-term modes of HPP on the criterion of minimizing the cost of electricity consumers in the wholesale market of Russia,” Energy, №11, pp. 39-41, 2013.

[5] B. Razykov, M. Dzhononov, S. Rasulov, F. Abdulloev, "Technical and economic evaluation of the efficiency of hydroelectric power stations in the market conditions of the power system," Bulletin of the Tajik Technical University, T. 4-4, №4, pp. 57-63, 2010.

[6] A.G. Rusina, Development of the theory and methodology of the analysis of electric power systems for control set the mode: dis. D.Sc., Tomsk, 2013, p. 297.

[7] T.A. Filippova, A.G. Rusina, "Modes of electric power stations and electric power systems," Proc. Guide the direction pf preparation «Power and Electrical Engineering», 2016 [Novosibirsk: Publishing House of Novosibirsk State Technical University, p. 400, 2016].

[8] E.A. Sovban, A.G. Rusina, J.K. Khujasaidov, T.A. Filippova, "Tasks of optimal performance of hydroelectric in power system," Proc. IFOST 2016, Pt. 2, pp. 251-254, 1-3 June 2016 [11 International forum on strategic technology: proc, Novosibirsk].

[9] A.G. Rusina, A.Y. Arestova, J.A. Frolov, P.A. Akulshina, E.A. Romanov, E.A. Sovban, D.H. Khujasaidov, The development, improvement and algorithmization principles address calculation of normal modes of electric power systems: Research report/executing, 2015 , p. 140. 
[10] A.G. Rusina, G.L. Rusin, D.H. Khujasaidov, (). "Features mode control grid Pamir: Electronics," Electrotechnology. Energy (EEE - 2015): Proc., Part 3, pp. 159-162, 9-12 June 2015 [Scientific. Tr. 7 Int. Scientific Conf. Young Scientists, 2015]. 